\title{
Construction and measurement of an electromagnetic noise generator based on an automotive coil
}

\author{
Marcelo Bender Perotoni*1, Samuel Nunes Nascimento Silva ${ }^{1}$ \\ ${ }^{1}$ Universidade Federal do ABC, Santo André, SP, Brazil
}

Received on April 03, 2017. Revised on May 09, 2017. Accepted on May 19, 2017.

\begin{abstract}
This article covers the design of an electromagnetic noise generator which uses an automotive coil as the main component. A short theoretical analysis covers the generation of high voltage pulsed current from the available DC source originated from the battery. An electronic dimmer, a circuit used to control the brightness of a light, by controlling the effective voltage that is delivered to the load, is used here to provide more degrees of freedom to the generated power as well as to insert higher order harmonics into the emitted electromagnetic spectrum. A prototype was built and its time domain current waveform is measured and shown, altogether with the measured radiated spectra, covering the frequency range from $20 \mathrm{~Hz}$ to $1 \mathrm{GHz}$. A broadband emission characteristics is verified, turning the use of common AM/FM radios impossible when close to the noise generator. A visible strong plasma is also seen due to the high generated voltages (around $60 \mathrm{kV}$ ).

Keywords: Applied Electromagnetism, Electromagnetic Compatibility.
\end{abstract}

\section{Introduction}

Electromagnetic interference effects were virtually unknown from the ordinary public unless sporadic cases such as television receivers showing annoying noises and video artifacts whenever a motorcycle passed by with a deficient shielding on its spark plug connection. With the widespread use of wireless systems and higher density electronic circuitry in household appliances, automobiles, arose the necessity of addressing proper EMC (Electromagnetic Compatibility) guidelines so that the mutual existence of all those systems are safeguarded. EMC is still absent from most Electrical Engineering and correlated academic programs though its effects have become well known in cases such as the mobile phone leaking its signal to nearby loudspeakers, which produces the famous beating sound in conferences or personal computer loudspeakers. The compliance to EMC guidelines is mandatory for several electronic systems nowadays, from regular domestic appliances to vehicular systems and biomedical instrumentation, otherwise their commercialization is not possible. So, it is of paramount importance for future professionals to be exposed to the EMC basic tenets during their academic training.

Besides those common EMC issues, from time to time mainstream media covers the problem of mobile phones found inside penitentiaries, illegally held by inmates. The solution is usually pointed out by experts - installation of a cell phone blocker, which in its simpler form generates an interfering signal on the same frequency range used

*Correspondence email address: mperoconsult@gmail.com by the mobile carriers (other systems operate with more sophisticated methods though). The signal is directed to be present only close to the penitentiary physical limits, as not to hinder ordinary users that pass by the local. The system operates analogously to two parties having a conversation in the middle of a loud and noisy nightclub; they must speak out louder and louder as to make themselves understood. In the case of mobile phones possessed by inmates, the phone control power system detects a larger carrier and tries to reach the base station with increasing transmitter power levels, which ends up draining out the battery capacity after a while.

This article covers an experimental system that generates intentional electromagnetic radiation noise, so that its effects can be seen on domestic appliances such as $\mathrm{AM} / \mathrm{FM}$ radios, computers, TV sets etc. It is based on an automotive coil, easily available and with low cost, and follows suit circuits presented on the internet as "High Voltage generators". The electromagnetic noise comes along with a plasma due to the ionized air across the coil terminals, which has a strong visual impact and presents applications in other areas of Physics besides $\mathrm{RF}$ /applied electromagnetics. The generation of electromagnetic noise from automotive spark system is not new, since 1974 issues concerning EMC are addressed [1], a problem that was alleviated by the inclusion of a resistor in series with the gap, a simple solution still in use today. A General Motors study [2] declared that the distributor system, with its long cables running all the way to the spark plugs are the most likely to generate electromagnetic noise in an automobile, reaching services such as the 
entertainment facilities and GPS, besides the traditional $\mathrm{AM} / \mathrm{FM}$ radios.

\section{Circuit modelling}

As the vehicle moves its motor shaft, an electric contact is periodically closed and opened, according to the simplified schematic shown in Fig. 1. With the switch $S$ closed, the coil $L$ has a current circulating due to the battery $V$,the capacitor operating as an open circuit due to the steady state current (infinite impedance). When the current flow is interrupted by opening the switch $S$ the current drops instantly to zero and a voltage is developed across the inductor terminals, as to keep the current circulating (counter electromotive force). Its primary reaches around 200-300V [3]. Since there is a transformation relation in terms of turns of the secondary over the primary, the secondary presents a magnified voltage to about 20 $\mathrm{kV}-40 \mathrm{kV}$, which is adequate to produce an explosion on the spark plug, inside the engine cylinder, with currents of 30 to $100 \mathrm{~mA}$, with low efficiencies - typically around $1 \%$ [4]. The capacitor $C$ in the schematic avoids the generation of arcs and high frequency spikes as the switches changes state, acting as a low-pass filter, short-circuiting high frequency signals and avoiding the coil current to be contaminated with these high frequency energy signals. The resistor $\mathrm{R}$ represents the ohmic wire losses on the coil and the EMI reduction resistance, artificially added to the spark plug.

With the switch $\mathrm{S}$ closed the inductor reaches the final steady-state current value:

$$
i=\frac{V}{R}
$$

The current charging in time domain follows the expression:

$$
i(t)=\frac{V}{R}\left(1-e^{-R t / L}\right)
$$

When the switch $S$ is opened, the capacitor $C$ configures the circuit as a typical series RLC, whose second order differential equation has a response in the format:

$$
i(t)=\frac{1}{s_{1}-s_{2}}\left(S_{1} e^{s_{1} t}-S_{2} e^{s_{2} t}\right)
$$

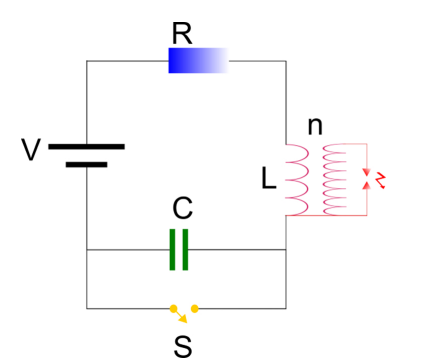

Figure 1: Simplified electric schematic of the automotive ignition system.
With the parameters $s_{1,2}$ defined as:

$$
s_{1,2}=\frac{-R}{L} \pm \sqrt{\left(\frac{R}{L}\right)^{2}-\frac{1}{L C}}
$$

Typical values for the circuit are [5] $V=12 \mathrm{~V}, R=2 \Omega$, $L=5 \mathrm{mH}$ and $C=10 \mu \mathrm{F}$, whose simulation in the time domain result is presented in fig. 2. It was considered the switch closed for $20 \mathrm{~ms}$, opened for $12.5 \mathrm{~ms}$ and then closed again. The high frequency oscillatory content of the pulse is seen after the $30 \mathrm{~ms}$ - when the stored energy is released on the RLC circuit.

The high voltage generated by the coil is applied to the spark plug, which has a small gap - open circuit, where the mixture (gas and air) has its dielectric breakdown broken and a spark is generated, detonating the explosive gas. The dielectric breakdown for the air is about $3 \mathrm{E} 6 \mathrm{~V} / \mathrm{m}$ [6], for instance. After the dielectric breakdown is broken there is a channel by which the electric current circulates (the air becomes a conductor through this channel). The time expression of this resistance is described by the Rompe-Weizel expression $[7,8]$. This expression is derived based on two assumptions: (i) the plasma conductivity is proportional to the internal energy balance of the system (instantaneous product of the voltage and current on the gap) and (ii) the relation between the plasma conductivity and the internal energy does not depend on time.

$$
r(t)=\frac{l}{\sqrt{\left(\frac{2 \alpha}{p}\right) \int_{0}^{t} i\left(t^{\prime}\right) d t^{\prime}}}
$$

Where $i$ is the current, $l$ the channel length, $\quad \alpha$ the spark coefficient which is determined by the atmospheric pressure $p$. The integral in the denominator imposes a low-pass filter effect to the current, which modulates the channel resistance.

\section{Electronic Circuit of the Coil Driver}

Since the coil operates as a self-transformer it requires a time-varying voltage according to the Lenz Law. The battery provides a DC (direct current) signal, so there is a need to impose a time-varying behavior. This switching imposed on the steady battery voltage is performed by the contact breaker, mechanically coupled to the rotating engine cam. Modern vehicular engines have electronic injection, whose solid-state circuits enable a better control on the correct spark plug firing, accordingly to factors such as plugs aging, type of combustible used, etc.

In principle, the simple connection of the household power AC signal (127 or $220 \mathrm{VAC}$ ) can generate the electromagnetic pulses and create the plasma (according to fig. 3) on an automobile coil. That means the dimmer circuit is optional - its goal is to provide a degree of freedom where the current conducting angle can be manually adjusted, therefore decreasing the power delivered 


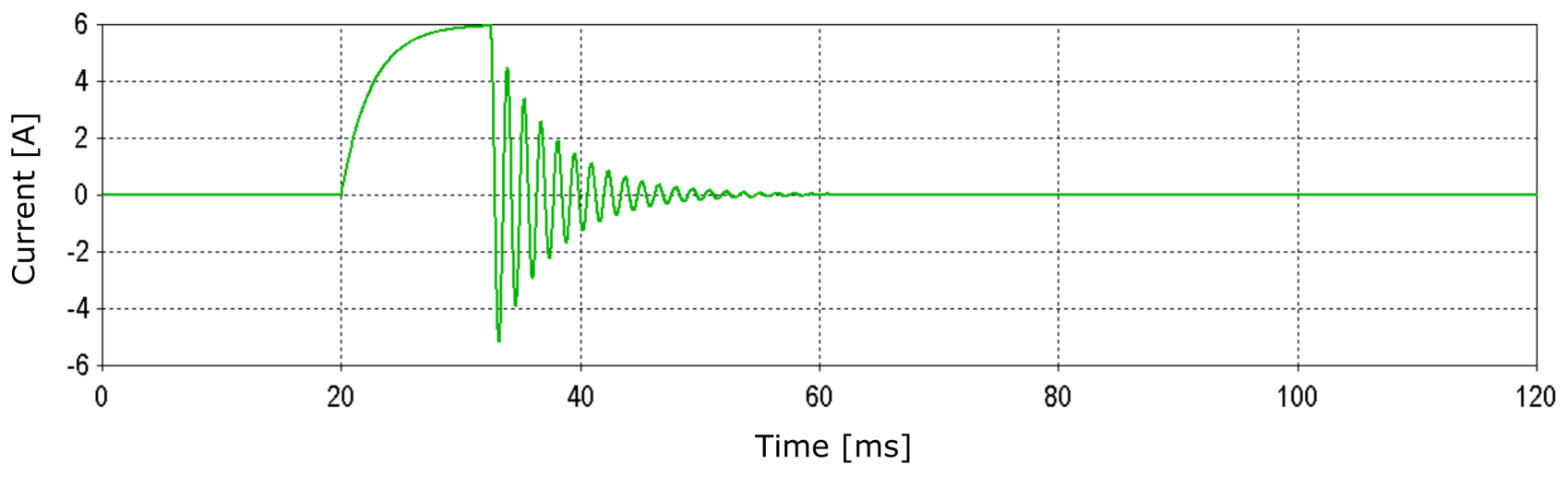

(a)

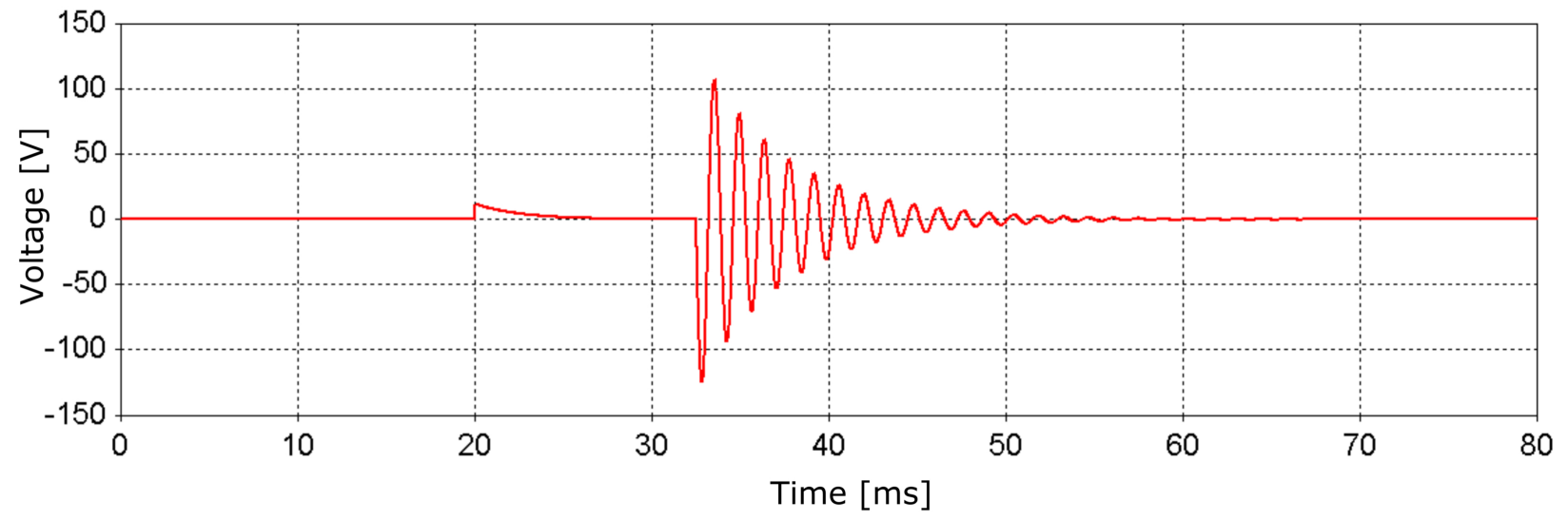

(b)

Figure 2: (a) Current on the coil primary and (b) its voltage, simulated results.

to the coil and also inserting higher frequency harmonics to the plasma current. The output voltage is extracted between the central coil and the negative terminal (the latter connected to its metallic body).

The complete dimmer circuit is presented on the fig. 4 , following the presented as a high voltage generator online [9]. The circuit is based on the three-terminal semiconductor switching device Triac, whose current circulating between the two anodes is controlled by the signal applied to its gate terminal. Once the threshold voltage is reached on the gate the device is turned on, allowing the current to flow. Triacs allow the current to flow in both directions, thereby controlling $\mathrm{AC}$ currents. By triggering the gate terminal in certain angles or phases of the $\mathrm{AC}$ sinusoidal current the amount of energy delivered to a load can be controlled. The dimmer circuit controls the trigger angle of the current by the Triac (TIC216B or similar). This angle is manually set by the user with the $220 \mathrm{~K} \Omega$ potentiometer, which controls the rate of charge of the $0.1 \mu \mathrm{F}$ capacitor. The circuit is simple and can be connected to $220 \mathrm{VAC}$ too, in this case the nominal voltage of the $3 \mu \mathrm{F}$ input capacitor should be chosen to be above $250 \mathrm{~V}$. The element named DB3 is a Diac (the name stands for "Diode $\mathrm{AC}$ "), and it conducts when

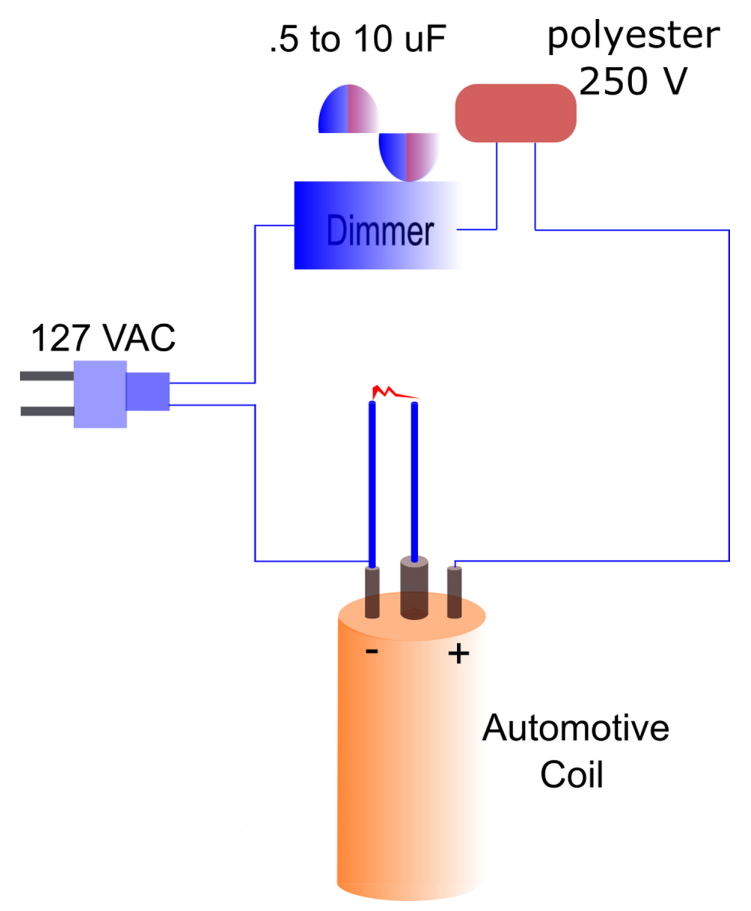

Figure 3: Electric schematic of the coil connected to the AC power outlet, by means of a dimmer and a polyester capacitor. 


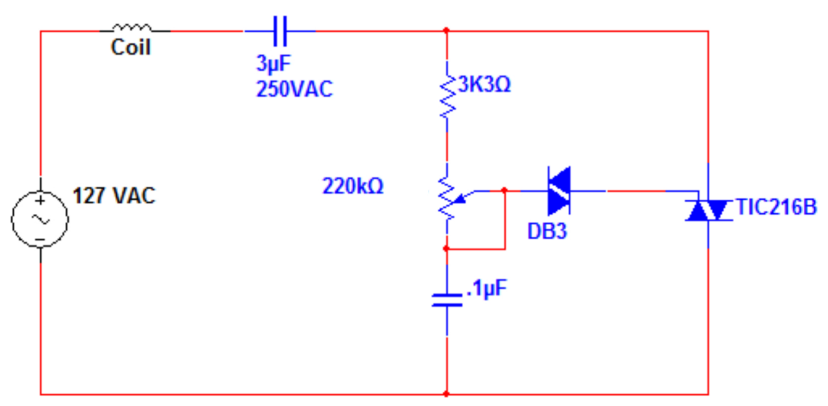

Figure 4: Electronic schematic of the Coil driver.

the voltage is larger than a fixed threshold; it does not possess a gate terminal like the triac.

Fig. 5 shows the circuit under test and a zoomed picture of its assembly. In virtue of the high voltages is important to ensure that all safety measures are properly followed - pliers with large isolation to handle the wires as well as discharging the capacitors and the coil after the circuit is disconnected from the AC mains - by shortcircuiting their respective terminals. Besides that, the generated plasma creates ozone (O3), detected due to its characteristic smell. Ozone is reported to be hazardous in high concentrations [10], so it is advisable to perform the tests in a ventilated area. The spark when connected to $220 \mathrm{VAC}$ is stronger than with $127 \mathrm{VAC}$, and its strength is accompanied not only by the ozone smell but also by a characteristic hum noise, due to the formed arc between the coil terminals.

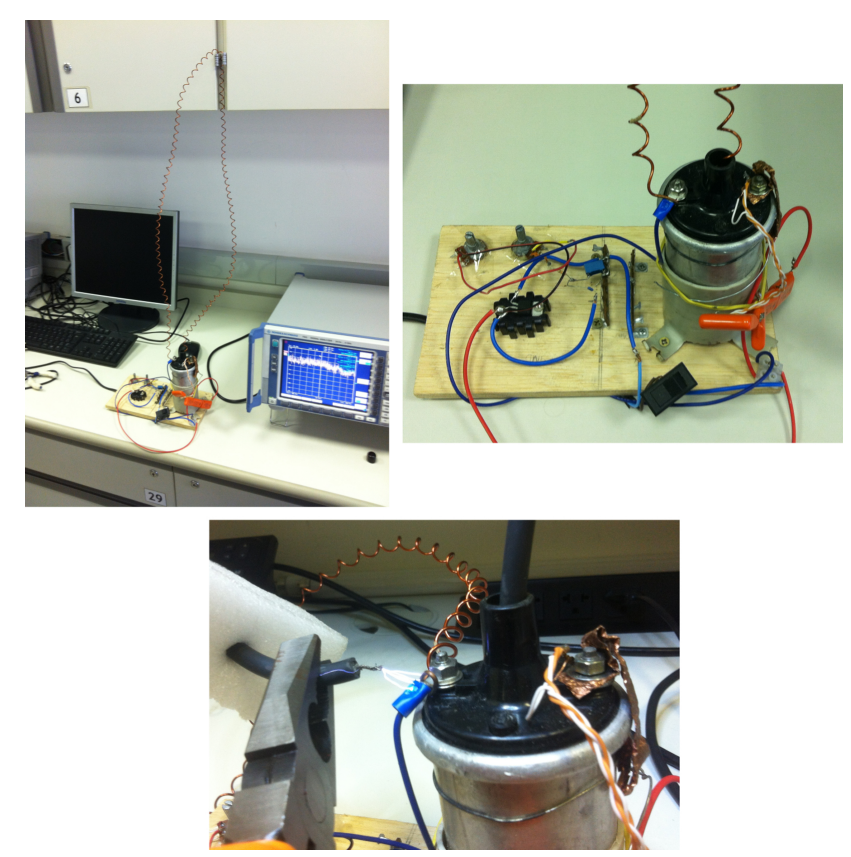

Figure 5: (Top left) coil and driver circuit under radiation test and (top right) zoomed vision of the assembly. Bottom, detail of the spark.

\section{Results}

The first topic of interest in the circuit is to determine the range of the coil secondary voltages. It is recommended to use an oscilloscope with isolated inputs. The isolated input oscilloscope as the name implies possesses a high isolation between the input ground (the terminal with small teeth) and the AC ground. Since the maximum input level of the used oscilloscope was $300 \mathrm{~V}$, a voltage divider resistor network was implemented, according to fig. 6. The resistors have large nominal values, as to conduct the minimum possible current, but needed to be covered with insulating tape, since without it the current jumps across their terminals instead of their bodies, after the air dielectric breakdown voltage is reached. Taking into consideration the resistors used the measured voltage can be expressed as:

$$
V_{\text {measured }}=\frac{39}{39+15,92.10^{3}} V_{\text {coil }}=2,44.10^{-3} V_{\text {coil }}
$$

Fig. 7 shows the captured waveforms in the oscilloscope. The periodic nature of the signal can be seen in fig. $7 \mathrm{a}$ whereas an isolated pulse is seen zoomed in on fig. $7 \mathrm{~b}$. According to eq.6, the secondary voltage can be estimated as $150 / 2.44 \mathrm{E}-3=61 \mathrm{kV}$, from a primary input of about $127 \mathrm{VAC}$.

Following the concept of Radiated Immunity [11] the radiated energy was measured with a signal analyzer Rohde and Schwarz FSQ, which covers the range of $20 \mathrm{~Hz}$ to $8 \mathrm{HGz}$. A simple $18 \mathrm{~cm}$ long telescopic antenna was used to pick up the electromagnetic radiation, located at about

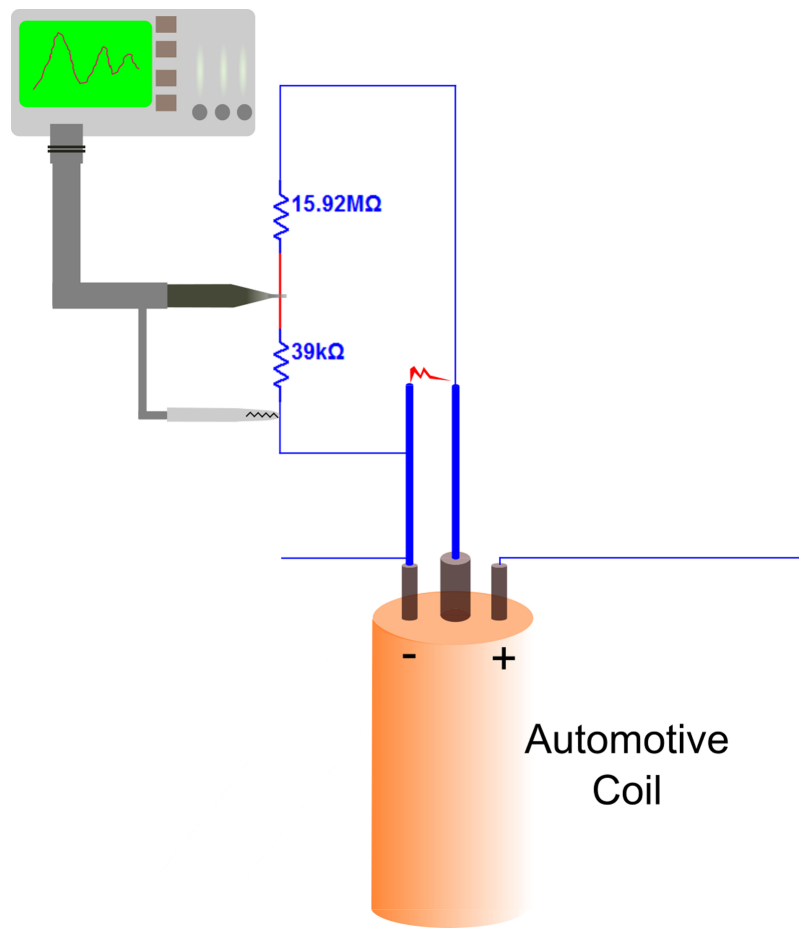

Figure 6: Resistor divider network used to measure the coil secondary voltage. 


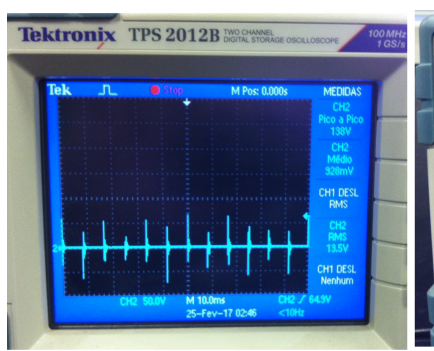

(a)

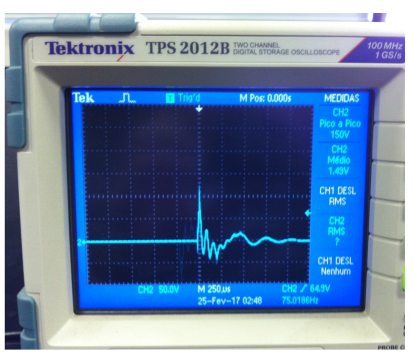

(b)
Figure 7: Time domain waveforms measured on the coil secondary for different time scales - (a) pulse train and (b) isolated pulse.

$1 \mathrm{~m}$ from the coil. This small antenna will receive less efficiently signals when it is electrically short, that means the inferior covered frequency range [12]. Since the system was measured in an ordinary laboratory environment, without any shielding and subjected to existing external signals, it was first measured the background noise. Fig. 8 shows the measurement results in the range of $20 \mathrm{~Hz}$ to $1 \mathrm{GHz}$, with the system connected to $127 \mathrm{VAC}$ mains outlet. It can be seen that the radiation covers the AM radio spectrum $(640 \mathrm{kHz}$ to $1 \mathrm{MHz}) ; \mathrm{FM}(88 \mathrm{MHz}$ to $108 \mathrm{MHz})$ as well as the Brazilian Digital TV $(470 \mathrm{MHz}-$ $806 \mathrm{MHz}$ ). The spectrum used by the cell phones (from about $824 \mathrm{MHz}$ to $900 \mathrm{MHz}$ ) showed that the coil radiation amplitude was close in amplitude to the existing mobile phone carriers. FM and AM radios operating in the area close to the coil produced no audible signal, only noise.

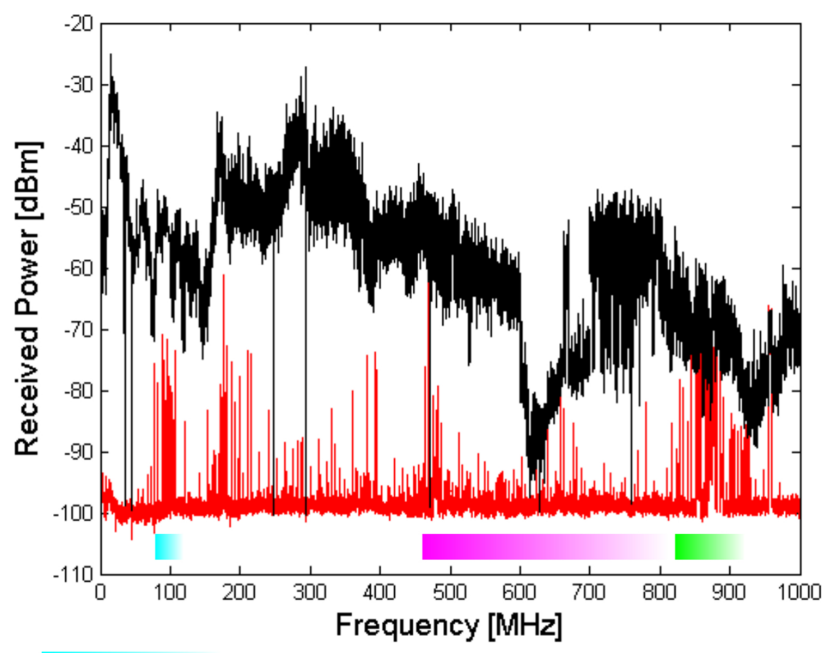

- M radio

Cellular DigitalTV

Figure 8: Measured radiation by the coil (black trace) and the existing spectrum (red trace).

\section{Conclusions}

This article covers the construction and measurement of an Electromagnetic Noise Generator based on an automotive coil. A short analysis of the simplified automotive electric circuit is presented, according to an RLCseries network. The peak voltage on the constructed coil secondary is measured altogether with its time-domain waveform, as well as the radiation electromagnetic energy covering the frequencies from $20 \mathrm{~Hz}$ to $1 \mathrm{GHz}$. The circuit can be used not only as a high voltage generator, as it is usually described in the internet, but also as a way to address electromagnetic interference effects with low cost and easily available components.

\section{References}

[1] R.R. Burgett, R.E. Massoll and D.R.V. Uum, IEEE Transactions on Electromagnetic Compatibility, 16, 160 (1974).

[2] V. Patel and M. Stefka, in: IEEE International Symposium on Electromagnetic Compatibility, Chicago, 2005, p. 19-23.

[3] Q. Deng, Y. Li, Y. Zhu, J. Yu and W. Quandi, in: WAC 2008 World Congress on Automation, Waikoloa, US, p. $1-4$.

[4] G.J. Rohwein, S.R. Babcook, M.T. Buttram and L.S. Camili, in: 10th IEEE International Pulsed Power Conference, 1995, Albuquerque, p. 40-46.

[5] G. Rizzoni, Fundamentals of Electrical Engineering (McGraw Hill, New York, 2009), 1st ed.

[6] P.A. Tipler, College Physics (Worth, New York, 1987).

[7] R. Rompe and W. Weizel, Z. Physilz 122, 636 (1944).

[8] I. Kang, O. Fugiwara and J. Wang, IEEE Electronic Letters 33, 1203 (1997).

[9] L.F. Netto, Geradores de Altas Tensões, 1999, disponível em http://www.feiradeciencias.com.br/sala15/15_ 37c.asp acessado em 29/03/2017.

[10] World Health Organization, Health Aspects of Air Pollution with Particulated Matter, Ozone and Nitrogen Dioxide (WHO, Bonn, 2003).

[11] C.R. Paul, Introduction to Electromagnetic Compatibility (Wiley Interscience, Hoboken, 2006), 2nd ed.

[12] C.A. Balanis, Antenna Theory - Analysis and Design (John Wiley \& Sons, New York, US, 1997), 2nd ed. 